\title{
Dyslipidemia and Related Risk Factors in a Saudi University Community
}

\author{
Fayez Hamam \\ Department of Pharmacology and Toxicology, College of Pharmacy, Taif University, Taif, Kingdom of Saudi Arabia \\ Email: fhamam@yahoo.com, f.hamam@tu.edu.sa
}

How to cite this paper: Hamam, F. (2017) Dyslipidemia and Related Risk Factors in a Saudi University Community. Food and $\mathrm{Nu}$ trition Sciences, 8, 56-69.

http://dx.doi.org/10.4236/fns.2017.81004

Received: November 26, 2016 Accepted: December 31, 2016 Published: January 3, 2017

Copyright $\odot 2017$ by author and Scientific Research Publishing Inc. This work is licensed under the Creative Commons Attribution International License (CC BY 4.0).

http://creativecommons.org/licenses/by/4.0/ (c) (i) Open Access

\begin{abstract}
Background/Objectives: Cardio vascular diseases (CVD) are considered a serious and prompt growing health problem in the Kingdom of Saudi Arabia, since it is the leading cause of morbidity and mortality. This study aimed to assess the prevalence of dyslipidemia and related risk factors among Health Sciences students in Taif University, KSA. Subjects/Methods: A sample of 80 students aged 17 - 26 years, were selected randomly from the Health Sciences colleges, Taif University. Participants were screened for blood lipid profile, obesity/overweight and related risk factors through filling pretested food frequency questionnaire. Anthropometric measurements and fasting blood samples were taken for determination of blood lipid profile, namely total cholesterol (TC), low density lipoprotein-cholesterol (LDL-c), high density lipoprotein-cholesterol (HDL-c), triacylglycerol (TAG) and the ratio of TC/ HDL-c. Results: The prevalence of hypercholesterolemia (TC $\geq 200 \mathrm{mg} / \mathrm{dl}$ ), hypertriglyceridemia (TAG $\geq 150 \mathrm{mg} / \mathrm{dl})$, high LDL-c $(\geq 130 \mathrm{mg} / \mathrm{dl})$, low HDL-c $(<40$ $\mathrm{mg} / \mathrm{dl}$ ) was $17.7 \%, 5.0 \%, 16.8 \%$, and $46.3 \%$, respectively. The overall prevalence of dyslipidemia was $60.0 \%$. Low HDL-c levels were the main prevalent dyslipidemia among participants. Overweight/obese students were more likely to show at least one undesirable lipid concentration. BMI, W_C, and academic level were significantly associated with unacceptable levels of HDL-c. TV viewing or computer/video games had significant effects on hypercholesterolemia, while only liver intake had significant impact on high TAG. Conclusions: Lipid study showed that dyslipidemia is very common and a significant health problem among university students of Taif University.
\end{abstract}

\section{Keywords}

Overweight, Obesity, Dyslipidemia, Food Habits, Lifestyle

\section{Introduction}

CVD (cardio vascular diseases) are the most important cause of morbidity and mortal- 
ity among the population of KSA [1]. In 2008, WHO reported that CVD resulted in death of 144 in every 100,000 residents live in the Kingdom. Thus, coronary heart disease (CHD) could be considered as one of the main health problems in KSA in terms of morbidity, mortality, and the economic cost of management of CVD [2].

Dyslipidemia was referred to as the occurrence of one or more of the following: TC $\geq$ $200 \mathrm{mg} / \mathrm{dl}$, LDL-c $\geq 130 \mathrm{mg} / \mathrm{dl}$, HDL-c $<40 \mathrm{mg} / \mathrm{dl}$, TAG $\geq 150 \mathrm{mg} / \mathrm{dl}$ and non-HDL-c $\geq$ $145 \mathrm{mg} / \mathrm{dl}$. Dyslipidemia is known as the major important adjustable risk variable for CVD. Reduction of incidence of CVD could be achieved via early diagnosis, diet and/or drug treatment. A clinical link was recognized between hyperlipidemia and new cases of heart disease [3] [4]. Several factors such as genetic susceptibility, elevated level of serum total cholesterol (TC), and a reduced level of high-density lipoprotein (HDLcholesterol) are the main risk factors related to $\mathrm{CHD}$ [5]. It is well known that obesity and dyslipidemia are positively correlated.

The prevalence of dyslipidemia was reported in several research works over the past twenty years in different locations of KSA and Gulf countries [4] [6] [7]. For instance, in a community-based study, the prevalence of high levels of cholesterol $(>6.2 \mathrm{mmol} / \mathrm{L})$ among Saudi males and females was $7 \%$, and $8 \%$, respectively. Furthermore, this study found as the age of participants increases, the prevalence of hypercholesterolemia increases accordingly. The prevalence of hypercholesterolemia for participants aged 40 to 59 years was $14 \%$ for males and $11 \%$ for females [4]. Additionally, this study showed a link between the rate of hypercholesterolemia and body mass index and smoking. Female subjects showed higher rate of hypercholesterolemia than males. However, this epidemiological study found that the prevalence of hypercholesterolemia among Saudi population is lower than their counterparts in Europe and America [4]. Al-Shehri et al. [6] reported that in Riyadh area (capital of KSA), 32.7\% of schoolchildren had high risk levels of TC, 33.1\% of students had LDL-c level in the high risk values, and $34.1 \%$ of participants had TAG level above desirable values. Furthermore, this study found that TAG levels increase with age in both sexes, in the meantime, level of LDL-c decreases with age, especially among schoolgirls. In a Kuwaiti university, the prevalence of high levels of blood lipids, overweight and obesity were $10.5 \%, 30.6 \%$ and $19.8 \%$, respectively. Moreover, the relationship between overweight/obesity as well as dyslipidemia was approved. Dyslipidemia was considerably higher among male students aged 18+ years than female counterparts or male students aged 18 years or less [8].

Therefore, CVD is considered a serious and prompt growing health problem in the Kingdom of Saudi Arabia, since it is the leading cause of morbidity and mortality. In adults, it is well known that the process of atherosclerosis underlying the CHD development starts early in life, therefore, the detection of those at higher risk of developing $\mathrm{CHD}$ at an early stage of life will be of great importance in the planning of prevention program. Determination of the dyslipidemia prevalence is crucial upon planning of health programs for primary as well as secondary prevention of CVD.

To the best of our knowledge this is the first human study conducted in a Saudi university community. It is a laboratory-, anthropometric- and questionnaire-based study. This study aimed to determine prevalence of dyslipidemia among male HSS, and to assess the effects of participants' dietary habits and lifestyle on blood lipid chemistry. It also investigated link between body mass index (BMI), waist circumference (W_C) and 
different blood lipid parameters. The outcomes of the current study will be the foundation of the second phase (an intervention study).

\section{Materials and Methods}

\subsection{Study Design and Study Population}

A cross-sectional study was carried out among HSS at Taif University in the period from July to December 2015. All students from Health Sciences campus were invited to contribute to this study. Eighty male students which constitute about $10 \%$ of students of Health Sciences colleges were randomly selected. Subjects were asked to fast at least 10 hours prior blood work. This study is different from other epidemiological studies since it included structured questionnaire, anthropometric measurements, and laboratory investigations.

The study tools:

A structured pre-examined questionnaire was organized into two main parts. The first part obtained information about demographic characters of respondents. This part also collected anthropometric measurements by the research team including height (meters), weight $(\mathrm{kg})$, and waist circumferences $(\mathrm{cm})$. Participants' height was determined to the closest $0.2 \mathrm{~cm}$ using a measuring scale equipped with sliding head part. Body mass was determined by calibrated digital weigh to the closest $0.1 \mathrm{~kg}$ after asking subjects to take off their shoes and heavy clothing if available. BMI was determined via standard equation [8]. The calculated BMIs were categorized to underweight, normal weight, overweight and obese (type I, II, and III). While cut off values for waist circumference (W_C) are as the following: if $\mathrm{W}_{-} \mathrm{C}<102 \mathrm{~cm}$, then the students are at lower risk of developing health problems, if $\mathrm{W}_{-} \mathrm{C} \geq 102 \mathrm{~cm}$, then students are at high risk [9]. The second part collected data about the lifestyle and eating habits among students.

\subsection{Blood Sampling and Laboratory Investigations}

Blood sample $(5 \mathrm{ml})$ was collected from each student after signing the consent form by certified health workers for determination of blood lipid profile. Participants in this study were kindly asked to fast at least ten hours before blood work. Serum was separated using centrifugation of blood for 3 - 5 minutes at $5000 \mathrm{rpm}$, and serum samples were stored at $-20^{\circ} \mathrm{C}$ till further analysis. Serum lipids were analyzed for complete lipid profile in a certified laboratory (Elaj Laboratories, Jeddah, KSA). Expected values for TC, TAG, HDL-c, and LDL-c were adapted from the American Heart Association [8]. The prevalence of dyslipidemia was determined using Venn diagram (Figure 1).

\subsection{Analysis of Data}

Data obtained were coded, entered and analyzed using the statistical package for social sciences (IBM SPSS, version 22, Armonk, NY: IBM Corp.). Means, frequencies and percentages were utilized to illustrate different parameters. Chi-square test was employed to determine the relationship between the students' demographic attributes, lifestyle, food habits and blood lipid profile. $P$-value $<0.05$ was considered significant. Relative standard deviations for the age, BMI and W_C were calculated using IBM-SPSS. 


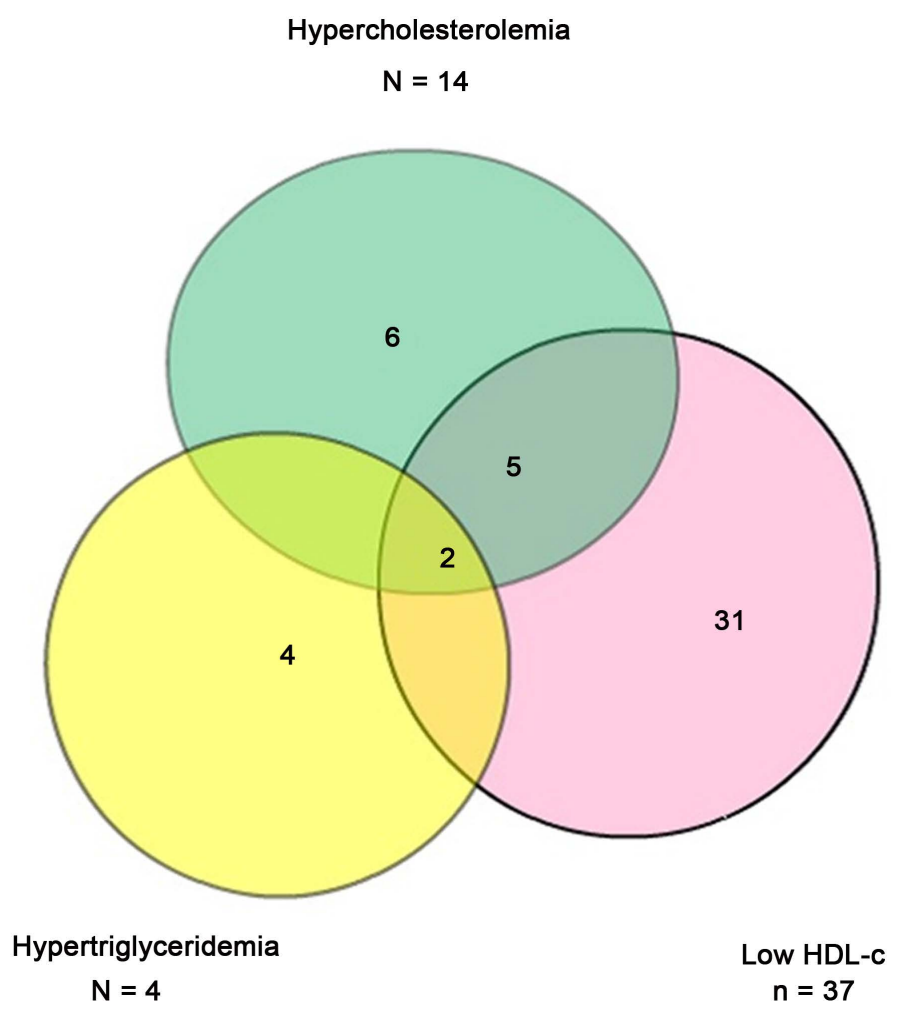

Figure 1. Venn diagram showed the three criteria used to determine dyslipidemia rate among Health Sciences students in a Saudi university community. The green circle represents subjects with hypercholesterolemia. The yellow circle represents university students with hypertriglyceridemia, while the pink circle represents students with low HDL-c.

\section{Results and Discussion}

\subsection{Effects of Student's Demographic Characteristics on Blood Lipid Profile}

A total of 80 university students participated in this study. Their Mean \pm SD age, BMI and W_C were $21.8 \pm 4.4,25.4 \pm 4.3$ and $82.3 \pm 12.8$, respectively. The prevalence of dyslipidemia, overweight, obesity and overweight/obesity were $60.0 \%, 33.8 \%, 16.3 \%$, and $50.1 \%$, respectively.

Blood lipid analysis revealed that, the means \pm SD of TAG, TC, HDL-c and LDL-c were $90.6 \pm 58.1,173.5 \pm 29.8,53.2 \pm 25.4$ and $105.0 \pm 27.0$, respectively. Majority 76 (95\%) of students had normal TAG level, although 14 (17.7\%) of them had borderline-high to high risk of TC levels. Regarding lipoproteins; only 43 (53.8\%) of participated students were among low risk category of HDL-c, while37 (46.3\%) of them were among moderate to high risk category. This means almost one in every two subjects had unfavorable level of HDL-c. Although less than half 38 (47.5\%) of students had normal level of LDL-c, but 14 (16.8\%) of students had borderline high to high risk level of LDL-c (Table 1). Ratio of TC/HDL-c indicated that 8 (10\%) of students were at high risk.

Table 2 illustrated the effects of BMI and W_C on blood lipid chemistry among participated students. It was found that, $27(33.8 \%)$ and 13 (16.3\%) of students overweight and obese respectively, and almost most of students 72 (94.7\%) among the no risk W_C 
Table 1. Lipid profile among male Health Sciences students at Taif University, KSA.

\begin{tabular}{|c|c|c|c|c|c|c|}
\hline \multirow{2}{*}{ Variables } & \multirow{2}{*}{ Mean \pm SD } & \multicolumn{4}{|c|}{ Classification } & \multirow{2}{*}{$\begin{array}{l}\text { Border-high } \\
\text { to high }\end{array}$} \\
\hline & & Normal & Boarder line risk & High risk & & \\
\hline Triacylglycerol (TAG) & $90.6 \pm 58.1$ & $76(95 \%)$ & $4(5 \%)$ & $0(0 \%)$ & & $4(5 \%)$ \\
\hline Total cholesterol (TC) & $173.5 \pm 29.8$ & $66(82.5 \%)$ & $12(15 \%)$ & $2(2.5 \%)$ & & $14(17.7 \%)$ \\
\hline High density & \multirow{2}{*}{$53.2 \pm 25.4$} & Low risk & Moderate risk & High risk & & \\
\hline lipoprotein (HDL-c) & & $43(53.8 \%)$ & $20(25 \%)$ & $17(21.3 \%)$ & & $37(46.3 \%)$ \\
\hline \multirow{2}{*}{$\begin{array}{c}\text { Low density } \\
\text { lipoprotein (LDL-c) }\end{array}$} & \multirow{2}{*}{$105.0 \pm 27.0$} & Normal & Near optimum & Boarder line risk & High risk & \\
\hline & & $38(47.5 \%)$ & $28(35 \%)$ & $11(13.8 \%)$ & $3(3.8 \%)$ & $14(16.8 \%)$ \\
\hline \multirow{2}{*}{ Ratio TC/HDL-c } & \multirow{2}{*}{$3.61 \pm 1.52$} & High protection & High risk & & & $8(10 \%)$ \\
\hline & & $72(90 \%)$ & $8(10 \%)$ & & & \\
\hline
\end{tabular}

According to the American Heart Association [1], expected values for total cholesterol as follow: Desirable when TC $<200 \mathrm{mg} / \mathrm{dl}$, borderline-high risk when TC 200 $239 \mathrm{mg} / \mathrm{dl}$, very high risk when TC > $200 \mathrm{mg} / \mathrm{dl}$. Expected values for triacylglycerol as the following: normal values when TAG < $150 \mathrm{mg} / \mathrm{dl}$, when TAG is the range $150-199 \mathrm{mg} / \mathrm{dl}$ then an individual is the borderline-high risk, if TAG is the range $200-499 \mathrm{mg} / \mathrm{dl}$ then he is in the high risk zone, when TAG level is higher than 500 $\mathrm{mg} / \mathrm{dl}$ then he is in the very high risk zone. Expected values for LDL-c as follow: desirable level when LDL-c $<100 \mathrm{mg} / \mathrm{dl}$, near optimal level when LDL-c is in the range $100-129 \mathrm{mg} / \mathrm{dl}$, if LDL-c is the range $130-159 \mathrm{mg} / \mathrm{dl}$, then an individual is the borderline-high risk, if LDL-c is the range $160-189 \mathrm{mg} / \mathrm{dl}$ then he is in the high risk zone, and finally if an individual's LDL-c is $190 \mathrm{mg} / \mathrm{dl}$ or above he is in the very high risk zone. Expected values for HDL-c as follow: if HDL-c level for a male is less than $40 \mathrm{mg} / \mathrm{dl}$ then he is in a major heart risk factor, while females will be in the same risk if their HDL-c level is less than $50 \mathrm{mg} / \mathrm{dl}$. If HDL-c level is higher than $60 \mathrm{mg} / \mathrm{dl}$, it mean some protection against heart disease for both sexes. Association, A.H. Understand Your Risk for High Cholesterol. 2016 [cited 2016 Nov. 3 , 2016 at 3:00 pm]; Available from: http://www.heart.org/HEARTORG/Conditions/Cholesterol/Cholesterol UCM 001089 SubHomePage.jsp.

Table 2. Effects of BMI \& W_C on blood lipid chemistry among male Health Sciences students at Taif University, KSA.

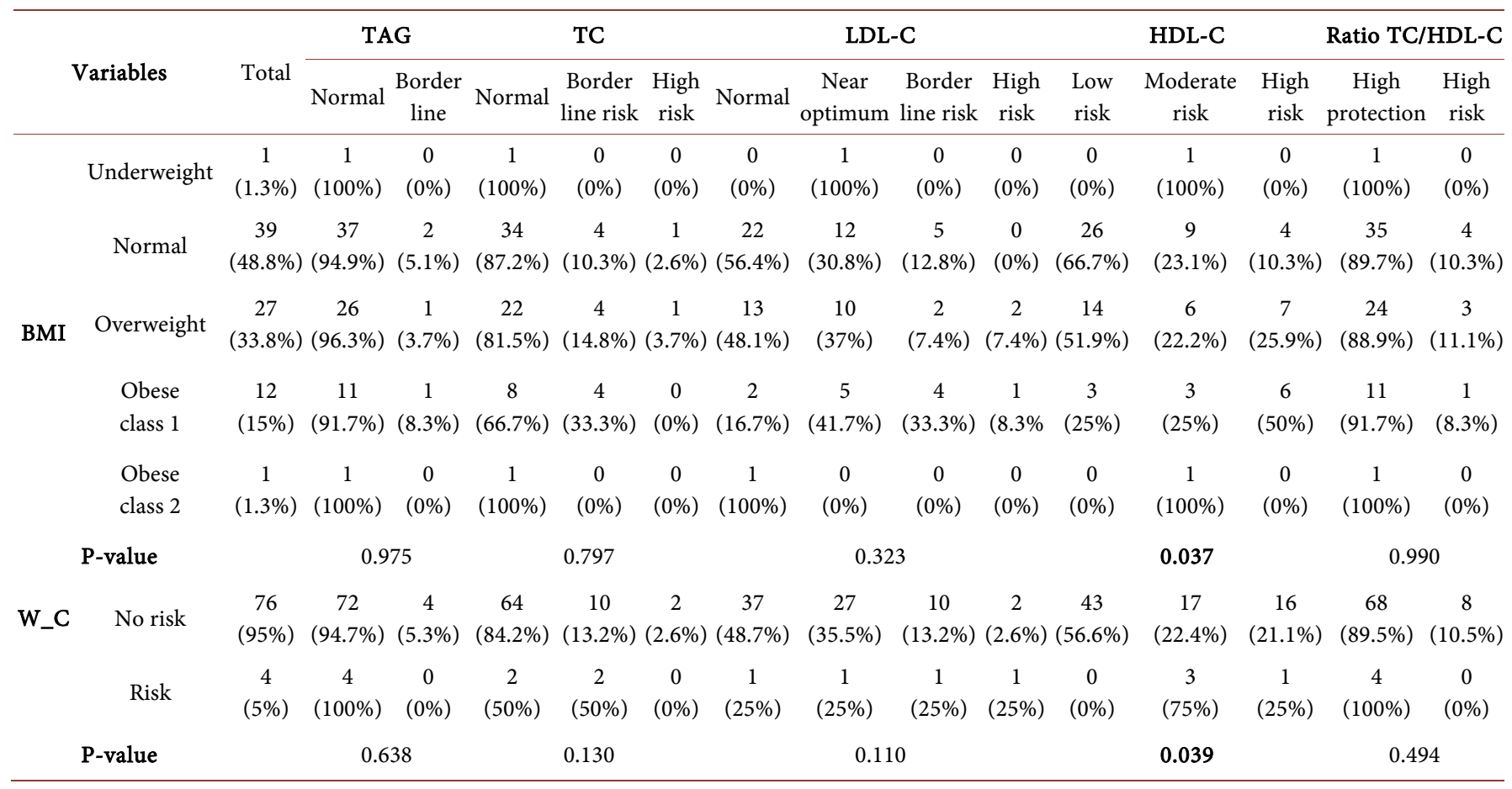

category. BMI and W_C had a significant effect on HDL-C. All students ranked under W_C risk category were among moderate or high risk of HDL-C classification ( $P=$ 0.039). On the other hand; $4(10.3 \%)$ of normal BMI students and $6(50 \%)$ of obese ones had high risk HDL-C levels $(P=0.037)$. 


\subsection{Effects of Demographic Characters on Blood Lipid Profile}

Table 3 showed that, the majority of students whom living with family had normal TAG 64 (94.1\%) and TC 55 (80.9\%), while only 30 (44.1\%) of them had normal LDL-c. there was no significant association between living with family and all lipid profile

Table 3. Effects of demographic characteristics on blood lipid profile among male Health Sciences students at Taif University, KSA.

\begin{tabular}{|c|c|c|c|c|c|c|c|c|c|c|c|c|c|c|c|c|}
\hline & \multirow[b]{2}{*}{ Variables } & \multirow[b]{2}{*}{ Total } & \multicolumn{2}{|c|}{ TAG } & \multicolumn{3}{|c|}{$\mathrm{TC}$} & \multicolumn{3}{|c|}{ HDL-C } & \multicolumn{3}{|c|}{ LDL-C } & \multicolumn{3}{|c|}{$\begin{array}{c}\text { Ratio } \\
\text { TC/HDL-C }\end{array}$} \\
\hline & & & Normal & $\begin{array}{l}\text { Border } \\
\text { line }\end{array}$ & Normal & $\begin{array}{l}\text { Border } \\
\text { line }\end{array}$ & $\begin{array}{c}\text { High } \\
\text { risk }\end{array}$ & $\begin{array}{l}\text { Low } \\
\text { risk }\end{array}$ & $\begin{array}{c}\text { Moderate } \\
\text { risk }\end{array}$ & $\begin{array}{l}\text { High } \\
\text { risk }\end{array}$ & Normal & $\begin{array}{c}\text { Near } \\
\text { optimum }\end{array}$ & $\begin{array}{c}\text { Border } \\
\text { line } \\
\text { risk }\end{array}$ & $\begin{array}{l}\text { High } \\
\text { risk }\end{array}$ & $\begin{array}{c}\text { High } \\
\text { protectior }\end{array}$ & $\begin{array}{l}\text { High } \\
\text { n risk }\end{array}$ \\
\hline \multirow{2}{*}{$\begin{array}{l}\text { Living } \\
\text { with } \\
\text { family }\end{array}$} & Yes & $\begin{array}{c}68 \\
(85 \%)\end{array}$ & $\begin{array}{c}64 \\
(94.1 \%)\end{array}$ & $\begin{array}{c}4 \\
(5.9 \%)\end{array}$ & $\begin{array}{c}55 \\
(80.9 \%)\end{array}$ & $\begin{array}{c}12 \\
(17.6 \%)\end{array}$ & $\begin{array}{c}1 \\
(1.5 \%)\end{array}$ & $\begin{array}{c}36 \\
(52.9 \%)\end{array}$ & $\begin{array}{c}17 \\
(25 \%)\end{array}$ & $\begin{array}{c}15 \\
(22.1 \%)\end{array}$ & $\begin{array}{c}30 \\
(44.1 \%)\end{array}$ & $\begin{array}{c}25 \\
(36.8 \%)\end{array}$ & $\begin{array}{c}11 \\
(16.2 \%)\end{array}$ & $\begin{array}{c}2 \\
(2.9 \%)\end{array}$ & $\begin{array}{c}60 \\
(88.2 \%)\end{array}$ & $\begin{array}{c}8 \\
(11.8 \%)\end{array}$ \\
\hline & No & $\begin{array}{c}12 \\
(15 \%)\end{array}$ & $\begin{array}{c}12 \\
(100 \%)\end{array}$ & $\begin{array}{c}0 \\
(0 \%)\end{array}$ & $\begin{array}{c}11 \\
(91.7 \%)\end{array}$ & $\begin{array}{c}0 \\
(0 \%)\end{array}$ & $\begin{array}{c}1 \\
(8.3 \%)\end{array}$ & $\begin{array}{c}7 \\
(58.3 \%)\end{array}$ & $\begin{array}{c}3 \\
(25 \%)\end{array}$ & $\begin{array}{c}2 \\
(16.7 \%)\end{array}$ & $\begin{array}{c}8 \\
(66.7 \%)\end{array}$ & $\begin{array}{c}3 \\
(25 \%)\end{array}$ & $\begin{array}{c}0 \\
(0 \%)\end{array}$ & $\begin{array}{c}1 \\
(8.3 \%)\end{array}$ & $\begin{array}{c}12 \\
(100 \%)\end{array}$ & $\begin{array}{c}0 \\
(0 \%)\end{array}$ \\
\hline \multirow{5}{*}{ 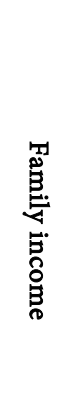 } & $\mathrm{P}$-value & & \multicolumn{2}{|c|}{0.389} & \multicolumn{3}{|c|}{0.124} & \multicolumn{3}{|c|}{0.907} & \multicolumn{4}{|c|}{0.238} & \multicolumn{2}{|c|}{0.210} \\
\hline & $<$ SAR 5000 & $\begin{array}{c}9 \\
(11.3 \%)\end{array}$ & $\begin{array}{c}8 \\
(88.9 \%)\end{array}$ & $\begin{array}{c}1 \\
(11.1 \%)\end{array}$ & $\begin{array}{c}6 \\
(66.7 \%)\end{array}$ & $\begin{array}{c}3 \\
(33.3 \%)\end{array}$ & $\begin{array}{c}0 \\
(0 \%)\end{array}$ & $\begin{array}{c}7 \\
(77.8 \%)\end{array}$ & $\begin{array}{c}1 \\
(11.1 \%)\end{array}$ & $\begin{array}{c}1 \\
(11.1 \%)\end{array}$ & $\begin{array}{c}6 \\
(66.7 \%)\end{array}$ & $\begin{array}{c}1 \\
(11.1 \%)\end{array}$ & $\begin{array}{c}2 \\
(22.2 \%)\end{array}$ & $\begin{array}{c}0 \\
(0 \%)\end{array}$ & $\begin{array}{c}8 \\
(88.9 \%)\end{array}$ & $\begin{array}{c}1 \\
(11.1 \%)\end{array}$ \\
\hline & $\begin{array}{c}\text { SAR } \\
5000-10,000\end{array}$ & $\begin{array}{c}20 \\
(25 \%)\end{array}$ & $\begin{array}{c}10 \\
(95 \%)\end{array}$ & $\begin{array}{c}1 \\
(5 \%)\end{array}$ & $\begin{array}{c}15 \\
(75 \%)\end{array}$ & $\begin{array}{c}4 \\
(20 \%)\end{array}$ & $\begin{array}{c}1 \\
(5 \%)\end{array}$ & $\begin{array}{c}10 \\
(50 \%)\end{array}$ & $\begin{array}{c}7 \\
(35 \%)\end{array}$ & $\begin{array}{c}3 \\
(15 \%)\end{array}$ & $\begin{array}{c}11 \\
(55 \%)\end{array}$ & $\begin{array}{c}4 \\
(20 \%)\end{array}$ & $\begin{array}{c}3 \\
(15 \%)\end{array}$ & $\begin{array}{c}2 \\
(10 \%)\end{array}$ & $\begin{array}{c}17 \\
(85 \%)\end{array}$ & $\begin{array}{c}3 \\
(15 \%)\end{array}$ \\
\hline & $\begin{array}{c}\text { SAR } \\
10,000-15,000\end{array}$ & $\begin{array}{c}19 \\
(23.8 \%)\end{array}$ & $\begin{array}{c}17 \\
(89.5 \%)\end{array}$ & $\begin{array}{c}2 \\
(10.5 \%)\end{array}$ & $\begin{array}{c}17 \\
(89.5 \%)\end{array}$ & $\begin{array}{c}1 \\
(5.3 \%)\end{array}$ & $\begin{array}{c}1 \\
(5.3 \%)\end{array}$ & $\begin{array}{c}10 \\
(52.6 \%)\end{array}$ & $\begin{array}{c}3 \\
(15.8 \%)\end{array}$ & $\begin{array}{c}6 \\
(31.6 \%)\end{array}$ & $\begin{array}{c}10 \\
(52.6 \%)\end{array}$ & $\begin{array}{c}7 \\
(36.8 \%)\end{array}$ & $\begin{array}{c}2 \\
(10.5 \%)\end{array}$ & $\begin{array}{c}0 \\
(0 \%)\end{array}$ & $\begin{array}{c}17 \\
(89.5 \%)\end{array}$ & $\begin{array}{c}2 \\
(10.5 \%)\end{array}$ \\
\hline & $>$ SAR 15,000 & $\begin{array}{c}32 \\
(40 \%)\end{array}$ & $\begin{array}{c}32 \\
(100 \%)\end{array}$ & $\begin{array}{c}0 \\
(0 \%)\end{array}$ & $\begin{array}{c}28 \\
(87.5 \%)\end{array}$ & $\begin{array}{c}4 \\
(12.5 \%)\end{array}$ & $\begin{array}{c}0 \\
(0 \%)\end{array}$ & $\begin{array}{c}16 \\
(50 \%)\end{array}$ & $\begin{array}{c}9 \\
(28.1 \%)\end{array}$ & $\begin{array}{c}7 \\
(21.9 \%)\end{array}$ & $\begin{array}{c}11 \\
(34.4 \%)\end{array}$ & $\begin{array}{c}16 \\
(50 \%)\end{array}$ & $\begin{array}{c}4 \\
(12.5 \%)\end{array}$ & $\begin{array}{c}1 \\
(3.1 \%)\end{array}$ & $\begin{array}{c}30 \\
(93.8 \%)\end{array}$ & $\begin{array}{c}2 \\
(6.3 \%)\end{array}$ \\
\hline \multirow{5}{*}{ 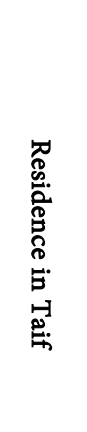 } & $\mathrm{P}$-value & & \multicolumn{2}{|c|}{0.306} & \multicolumn{3}{|c|}{0.379} & \multicolumn{3}{|c|}{0.527} & \multicolumn{4}{|c|}{0.285} & \multicolumn{2}{|c|}{0.783} \\
\hline & North & $\begin{array}{c}17 \\
(21.3 \%)\end{array}$ & $\begin{array}{c}17 \\
(100 \%)\end{array}$ & $\begin{array}{c}0 \\
(0 \%)\end{array}$ & $\begin{array}{c}16 \\
(94.1 \%)\end{array}$ & $\begin{array}{c}1 \\
(5.9 \%)\end{array}$ & $\begin{array}{c}0 \\
(0 \%)\end{array}$ & $\begin{array}{c}12 \\
(70.6 \%)\end{array}$ & $\begin{array}{c}4 \\
(23.5 \%)\end{array}$ & $\begin{array}{c}1 \\
(5.9 \%)\end{array}$ & $\begin{array}{c}12 \\
(70.6 \%)\end{array}$ & $\begin{array}{c}3 \\
(17.6 \%)\end{array}$ & $\begin{array}{c}2 \\
(11.8 \%)\end{array}$ & $\begin{array}{c}0 \\
(0 \%)\end{array}$ & $\begin{array}{c}17 \\
(100 \%)\end{array}$ & $\begin{array}{c}0 \\
(0 \%)\end{array}$ \\
\hline & South & $\begin{array}{c}26 \\
(32.5 \%)\end{array}$ & $\begin{array}{c}26 \\
(100 \%)\end{array}$ & $\begin{array}{c}0 \\
(0 \%)\end{array}$ & $\begin{array}{c}22 \\
(84.6 \%)\end{array}$ & $\begin{array}{c}4 \\
(15.4 \%)\end{array}$ & $\begin{array}{c}0 \\
(0 \%)\end{array}$ & $\begin{array}{c}12 \\
(46.2 \%)\end{array}$ & $\begin{array}{c}7 \\
(26.9 \%)\end{array}$ & $\begin{array}{c}7 \\
(26.9 \%)\end{array}$ & $\begin{array}{c}11 \\
(42.3 \%)\end{array}$ & $\begin{array}{c}11 \\
(42.3 \%)\end{array}$ & $\begin{array}{c}3 \\
(11.5 \%)\end{array}$ & $\begin{array}{c}1 \\
(3.8 \%)\end{array}$ & $\begin{array}{c}23 \\
(88.5 \%)\end{array}$ & $\begin{array}{c}3 \\
(11.5 \%)\end{array}$ \\
\hline & East & $\begin{array}{c}21 \\
(26.3 \%)\end{array}$ & $\begin{array}{c}18 \\
(85.7 \%)\end{array}$ & $\begin{array}{c}3 \\
(14.3 \%)\end{array}$ & $\begin{array}{c}16 \\
(76.2 \%)\end{array}$ & $\begin{array}{c}4 \\
(19 \%)\end{array}$ & $\begin{array}{c}1 \\
(4.8 \%)\end{array}$ & $\begin{array}{c}9 \\
(42.9 \%)\end{array}$ & $\begin{array}{c}6 \\
(28.6 \%)\end{array}$ & $\begin{array}{c}6 \\
(28.6 \%)\end{array}$ & $\begin{array}{c}7 \\
(33.3 \%)\end{array}$ & $\begin{array}{c}10 \\
(47.6 \%)\end{array}$ & $\begin{array}{c}3 \\
(14.3 \%)\end{array}$ & $\begin{array}{c}1 \\
(4.8 \%)\end{array}$ & $\begin{array}{c}18 \\
(85.7 \%)\end{array}$ & $\begin{array}{c}3 \\
(14.3 \%)\end{array}$ \\
\hline & West & $\begin{array}{c}16 \\
(20 \%)\end{array}$ & $\begin{array}{c}15 \\
(93.8 \%)\end{array}$ & $\begin{array}{c}1 \\
(6.3 \%)\end{array}$ & $\begin{array}{c}12 \\
(75 \%)\end{array}$ & $\begin{array}{c}3 \\
(18.8 \%)\end{array}$ & $\begin{array}{c}1 \\
(6.3 \%)\end{array}$ & $\begin{array}{c}10 \\
(62.5 \%)\end{array}$ & $\begin{array}{c}3 \\
(18.8 \%)\end{array}$ & $\begin{array}{c}3 \\
(18.8 \%)\end{array}$ & $\begin{array}{c}8 \\
(50 \%)\end{array}$ & $\begin{array}{c}4 \\
(25 \%)\end{array}$ & $\begin{array}{c}3 \\
(18.8 \%)\end{array}$ & $\begin{array}{c}1 \\
(6.3 \%)\end{array}$ & $\begin{array}{c}14 \\
(87.5 \%)\end{array}$ & $\begin{array}{c}2 \\
(12.5 \%)\end{array}$ \\
\hline \multirow{7}{*}{ 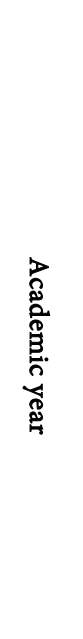 } & $P$-value & & \multicolumn{2}{|c|}{0.106} & \multicolumn{3}{|c|}{0.645} & \multicolumn{3}{|c|}{0.526} & \multicolumn{4}{|c|}{0.571} & \multicolumn{2}{|c|}{0.476} \\
\hline & 1st year & $\begin{array}{c}13 \\
(16.3 \%)\end{array}$ & $\begin{array}{c}13 \\
(100 \%)\end{array}$ & $\begin{array}{c}0 \\
(0 \%)\end{array}$ & $\begin{array}{c}11 \\
(84.6 \%)\end{array}$ & $\begin{array}{c}2 \\
(15.4 \%)\end{array}$ & $\begin{array}{c}0 \\
(0 \%)\end{array}$ & $\begin{array}{c}4 \\
(30.8 \%)\end{array}$ & $\begin{array}{c}6 \\
(46.2 \%)\end{array}$ & $\begin{array}{c}3 \\
(23.1 \%)\end{array}$ & $\begin{array}{c}7 \\
(53.8 \%)\end{array}$ & $\begin{array}{c}5 \\
(38.5 \%)\end{array}$ & $\begin{array}{c}1 \\
(7.7 \%)\end{array}$ & $\begin{array}{c}0 \\
(0 \%)\end{array}$ & $\begin{array}{c}13 \\
(100 \%)\end{array}$ & $\begin{array}{c}0 \\
(0 \%)\end{array}$ \\
\hline & 2nd Year & $\begin{array}{c}9 \\
(11.3 \%)\end{array}$ & $\begin{array}{c}9 \\
(100 \%)\end{array}$ & $\begin{array}{c}0 \\
(0 \%)\end{array}$ & $\begin{array}{c}9 \\
(100 \%)\end{array}$ & $\begin{array}{c}0 \\
(0 \%)\end{array}$ & $\begin{array}{c}0 \\
(0 \%)\end{array}$ & $\begin{array}{c}7 \\
(77.8 \%)\end{array}$ & $\begin{array}{c}1 \\
(11.1 \%)\end{array}$ & $\begin{array}{c}1 \\
(11.1 \%)\end{array}$ & $\begin{array}{c}5 \\
(55.6 \%)\end{array}$ & $\begin{array}{c}4 \\
(44.4 \%)\end{array}$ & $\begin{array}{c}0 \\
(0 \%)\end{array}$ & $\begin{array}{c}0 \\
(0 \%)\end{array}$ & $\begin{array}{c}8 \\
(88.9 \%)\end{array}$ & $\begin{array}{c}1 \\
(11.1 \%)\end{array}$ \\
\hline & 3 rd year & $\begin{array}{c}21 \\
(26.3 \%)\end{array}$ & $\begin{array}{c}19 \\
(90.5 \%)\end{array}$ & $\begin{array}{c}2 \\
(9.5 \%)\end{array}$ & $\begin{array}{c}19 \\
(0.5 \%)\end{array}$ & $\begin{array}{c}2 \\
(9.5 \%)\end{array}$ & $\begin{array}{c}0 \\
(0 \%)\end{array}$ & $\begin{array}{c}13 \\
(61.9 \%\end{array}$ & $\begin{array}{c}5 \\
(23.8 \%)\end{array}$ & $\begin{array}{c}3 \\
(14.3 \%)\end{array}$ & $\begin{array}{c}11 \\
(52.4 \%)\end{array}$ & $\begin{array}{c}8 \\
(38.1 \%)\end{array}$ & $\begin{array}{c}2 \\
(9.5 \%)\end{array}$ & $\begin{array}{c}0 \\
(0 \%)\end{array}$ & $\begin{array}{c}19 \\
(90.5 \%)\end{array}$ & $\begin{array}{c}2 \\
(9.5 \%)\end{array}$ \\
\hline & 4th Year & $\begin{array}{c}13 \\
(16.3 \%)\end{array}$ & $\begin{array}{c}13 \\
(100 \%)\end{array}$ & $\begin{array}{c}0 \\
(0 \%)\end{array}$ & $\begin{array}{c}12 \\
(92.3 \%)\end{array}$ & $\begin{array}{c}1 \\
(7.7 \%)\end{array}$ & $\begin{array}{c}0 \\
(0 \%)\end{array}$ & $\begin{array}{c}11 \\
(84.6 \%)\end{array}$ & $\begin{array}{c}0 \\
(0 \%)\end{array}$ & $\begin{array}{c}2 \\
(15.4 \%)\end{array}$ & $\begin{array}{c}8 \\
(61.5 \%)\end{array}$ & $\begin{array}{c}3 \\
(23.1 \%)\end{array}$ & $\begin{array}{c}2 \\
(15.4 \%)\end{array}$ & $\begin{array}{c}0 \\
(0 \%)\end{array}$ & $\begin{array}{c}12 \\
(92.3 \%)\end{array}$ & $\begin{array}{c}1 \\
(7.7 \%)\end{array}$ \\
\hline & 5th Year & $\begin{array}{c}21 \\
(26.3 \%)\end{array}$ & $\begin{array}{c}20 \\
(95.2 \%)\end{array}$ & $\begin{array}{c}1 \\
(4.8 \%)\end{array}$ & $\begin{array}{c}14 \\
(66.7 \%)\end{array}$ & $\begin{array}{c}5 \\
(23.8 \%)\end{array}$ & $\begin{array}{c}2 \\
(9.5 \%)\end{array}$ & $\begin{array}{c}8 \\
(38.1 \%)\end{array}$ & $\begin{array}{c}6 \\
(28.6 \%)\end{array}$ & $\begin{array}{c}7 \\
(33.3 \%)\end{array}$ & $\begin{array}{c}6 \\
(28.6 \%)\end{array}$ & $\begin{array}{c}8 \\
(38.1 \%)\end{array}$ & $\begin{array}{c}5 \\
(23.8 \%)\end{array}$ & $\begin{array}{c}2 \\
(9.5 \%)\end{array}$ & $\begin{array}{c}18 \\
(85.7 \%)\end{array}$ & $\begin{array}{c}3 \\
(14.3 \%)\end{array}$ \\
\hline & 6th year & $\begin{array}{c}3 \\
(3.8 \%)\end{array}$ & $\begin{array}{c}2 \\
(66.7 \%)\end{array}$ & $\begin{array}{c}1 \\
(33.3 \%)\end{array}$ & $\begin{array}{c}1 \\
(33.3 \%)\end{array}$ & $\begin{array}{c}2 \\
(66.7 \%)\end{array}$ & $\begin{array}{c}0 \\
(0 \%)\end{array}$ & $\begin{array}{c}0 \\
(0 \%)\end{array}$ & $\begin{array}{c}0 \\
(0 \%)\end{array}$ & $\begin{array}{c}1 \\
(33.3 \%)\end{array}$ & $\begin{array}{c}1 \\
(33.3 \%)\end{array}$ & $\begin{array}{c}0 \\
(0 \%)\end{array}$ & $\begin{array}{c}1 \\
(33.3 \%)\end{array}$ & $\begin{array}{c}1 \\
(33.3 \%)\end{array}$ & $\begin{array}{c}2 \\
(66.7 \%)\end{array}$ & $\begin{array}{c}1 \\
(33.3 \%)\end{array}$ \\
\hline & $\mathrm{P}$-value & & 0.1 & 166 & & 0.087 & & & 0.045 & & & 0.18 & & & 0.5 & 581 \\
\hline
\end{tabular}


analysis $(P>0.05)$.There was a significant difference between students' academic levels and their HDL-c $(P=0.045)$. Students had low risk HDL-c from 1st years level were lower $4(30.8 \%)$ than those from second year 7 (77.8\%) and from fourth year students $11(84.6 \%)$. Other demographic characters and their effects on lipid profile are declared in Table 3.

\subsection{Effects of Life Style on Blood Lipid Profile}

Although physical activity and doing exercise has an important effects on lipid level, overall 33 (41.3\%) of participants did not exercise through their days. However, this effect was insignificant $(P>0.05)$. While watching TV or using computer had a significant effect on TC level $(P=0.043)$. Normal TC level was found higher among students spent shorter time on TV or computers usage. On the other hand, neither hours of night nor daylight sleep had a significant effect on lipid profile $(P>0.05)$. Table 4 showed that $28(35 \%)$ of students sleep more than 4 hours at daylight and $10(12.5 \%)$ of them sleep less than 4 hours at night.

\subsection{Effects of Eating Habits and Selected Food Intake on Blood Lipid Profile}

Table 5 and Table 6 illustrated that, in this study eating habits and most of investigated food intake had insignificant effects on lipid profile $(P>0.05)$. In Table 5 , the results revealed that, numerous number of students had snacks as apart from regular meals either daily $18(22.5 \%)$ or 4 - 6 times weekly 17 (21.3\%). Drinking sugar-sweetened carbonated beverages is a common habit among youth. One fourth 20 (25\%) of investigated students drink one or more cans of soft drink daily. Table 6 elucidates selected food intake habits among male students. It found that, 27 (33.8\%) out of students had ate meat daily, while $10(12.5 \%)$ of them drink milk daily and $43(53.8 \%)$ of students consumed sea-food or fish only 1 - 2 times per month.

Serum TAG levels were normal in $95 \%$ of students, while only $5 \%$ of them either located within the borderline-high to high risk, which was much lower than Al-Shehri et al. [6] who showed that $34.1 \%$ of schoolchildren in KSA had TAG level above desirable values. Hypertriglyceridemia prevalence (5\%) among HSS was close to their counterparts in a Kuwaiti university community (8.7\%) [8]. In Egypt, Abdel Wahed et al. [10] found that $29.7 \%$ of college students had hypertriglyceridemia. Additionally, in KSA $33.6 \%$ of female college students had unacceptable level of triacylglycerol [11]. Results of this study indicated that hypertriglyceridemia rate (10.2\%) was half of world's adult population aged 15 to 65 years old [12].

The prevalence of hypercholesterolemia (>200 $\mathrm{mg} / \mathrm{dl}$ ) was $17.7 \%$ which was almost the double findings of Al-Nuaim et al. [4] who found that the prevalence of high TC among Saudi male and female schoolchildren were $7 \%$, and $8 \%$, respectively. Our figures $(17.7 \%)$ appear to be low particularly when comparing them to similar studies carried out on this age group. In a university-based studies conducted among several Arab countries, the prevalence of hypercholesterolemia ranged from about $27 \%$ to $38 \%$ [10] [11] [13] [14] [15]. However, in a Kuwaiti university, the prevalence of hypercholesterolemia (2.3\%) was much lower than other studies and our results [16]. In a community- 
Table 4. Effects of selected life style on blood lipid profile among male Health Sciences students at Taif University, KSA.

\begin{tabular}{|c|c|c|c|c|c|c|c|c|c|c|c|c|c|c|c|c|}
\hline \multirow{2}{*}{\multicolumn{2}{|c|}{ Variables }} & \multirow[b]{2}{*}{ Total } & \multicolumn{2}{|c|}{ TAG } & \multicolumn{3}{|c|}{ TC } & \multicolumn{3}{|c|}{ HDL-C } & \multicolumn{3}{|c|}{ LDL-C } & \multicolumn{3}{|c|}{ Ratio TC/HDL-C } \\
\hline & & & Normal & $\begin{array}{l}\text { Border } \\
\text { line }\end{array}$ & Normal & $\begin{array}{c}\text { Border } \\
\text { line }\end{array}$ & $\begin{array}{c}\text { High } \\
\text { risk }\end{array}$ & $\begin{array}{l}\text { Low } \\
\text { risk }\end{array}$ & $\begin{array}{c}\text { Moderate } \\
\text { risk }\end{array}$ & $\begin{array}{l}\text { High } \\
\text { risk }\end{array}$ & Normal & $\begin{array}{c}\text { Near } \\
\text { optimum }\end{array}$ & $\begin{array}{l}\text { Border } \\
\text { line } \\
\text { risk }\end{array}$ & $\begin{array}{l}\text { High } \\
\text { risk }\end{array}$ & $\begin{array}{c}\text { High } \\
\text { protection }\end{array}$ & $\begin{array}{l}\text { High } \\
\text { risk }\end{array}$ \\
\hline \multirow{2}{*}{$\begin{array}{l}\text { Physical } \\
\text { activity } \\
\text { and doing } \\
\text { exercise }\end{array}$} & Yes & $\begin{array}{c}47 \\
(58.8 \%)\end{array}$ & $\begin{array}{c}46 \\
(97.9 \%)\end{array}$ & $\begin{array}{c}1 \\
(2.1 \%)\end{array}$ & $\begin{array}{c}38 \\
(80.9 \%)\end{array}$ & $\begin{array}{c}9 \\
(19.1 \%)\end{array}$ & $\begin{array}{c}0 \\
(0 \%)\end{array}$ & $\begin{array}{c}27 \\
(57.4 \%)\end{array}$ & $\begin{array}{c}12 \\
(25.5 \%)\end{array}$ & $\begin{array}{c}8 \\
(17 \%)\end{array}$ & $\begin{array}{c}22 \\
(46.8 \%)\end{array}$ & $\begin{array}{c}16 \\
(34 \%)\end{array}$ & $\begin{array}{c}8 \\
(17 \%)\end{array}$ & $\begin{array}{c}1 \\
(2.1 \%)\end{array}$ & $\begin{array}{c}44 \\
(93.6 \%)\end{array}$ & $\begin{array}{c}3 \\
(6.4 \%)\end{array}$ \\
\hline & No & $\begin{array}{c}33 \\
(41.3 \%)\end{array}$ & $\begin{array}{c}30 \\
(90.9 \%)\end{array}$ & $\begin{array}{c}3 \\
(9.1 \%)\end{array}$ & $\begin{array}{c}28 \\
(84.8 \%)\end{array}$ & $\begin{array}{c}3 \\
(9.1 \%)\end{array}$ & $\begin{array}{c}2 \\
(6.1 \%)\end{array}$ & $\begin{array}{c}16 \\
(48.5 \%)\end{array}$ & $\begin{array}{c}8 \\
(24.2 \%)\end{array}$ & $\begin{array}{c}9 \\
(27.3 \%)\end{array}$ & $\begin{array}{c}16 \\
(48.5 \%)\end{array}$ & $\begin{array}{c}12 \\
(36.4 \%)\end{array}$ & $\begin{array}{c}3 \\
(9.1 \%)\end{array}$ & $2(6.1 \%)$ & $\begin{array}{c}28 \\
(84.8 \%\end{array}$ & $\begin{array}{c}5 \\
(15.2 \%)\end{array}$ \\
\hline \multicolumn{2}{|c|}{$\mathrm{P}$-value } & \multicolumn{3}{|c|}{0.159} & \multicolumn{3}{|c|}{0.123} & \multicolumn{3}{|c|}{0.532} & \multicolumn{4}{|c|}{0.631} & \multicolumn{2}{|c|}{0.198} \\
\hline \multirow{4}{*}{ 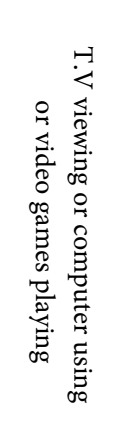 } & $<2 \mathrm{hrs}$ & $\begin{array}{c}2 \\
(2.3)\end{array}$ & $\begin{array}{c}2 \\
(100 \%)\end{array}$ & $\begin{array}{c}0 \\
(0 \%)\end{array}$ & $\begin{array}{c}1 \\
(50 \%)\end{array}$ & $\begin{array}{c}1 \\
(50 \%)\end{array}$ & $\begin{array}{c}0 \\
(0 \%)\end{array}$ & $\begin{array}{c}1 \\
(50 \%)\end{array}$ & $\begin{array}{c}1 \\
(50 \%)\end{array}$ & $\begin{array}{c}0 \\
(0 \%)\end{array}$ & $\begin{array}{c}1 \\
(50 \%)\end{array}$ & $\begin{array}{c}0 \\
(0 \%)\end{array}$ & $\begin{array}{c}1 \\
(50 \%)\end{array}$ & $\begin{array}{c}0 \\
(0 \%)\end{array}$ & $\begin{array}{c}2 \\
(100 \%)\end{array}$ & $\begin{array}{c}0 \\
(0 \%)\end{array}$ \\
\hline & $\begin{array}{l}2-4 \\
\text { hrs }\end{array}$ & $\begin{array}{c}14 \\
(17.5 \%)\end{array}$ & $\begin{array}{c}13 \\
(92.9 \%)\end{array}$ & $\begin{array}{c}1 \\
(7.1 \%)\end{array}$ & $\begin{array}{c}9 \\
(64.3 \%)\end{array}$ & $\begin{array}{c}5 \\
(35.7 \%)\end{array}$ & $\begin{array}{c}0 \\
(0 \%)\end{array}$ & $\begin{array}{c}8 \\
(57.1 \%)\end{array}$ & $\begin{array}{c}1 \\
(7.1 \%)\end{array}$ & $\begin{array}{c}5 \\
(35.7 \%)\end{array}$ & $\begin{array}{c}4 \\
(28.6 \%)\end{array}$ & $\begin{array}{c}6 \\
(42.9 \%)\end{array}$ & $\begin{array}{c}3 \\
(21.4 \%)\end{array}$ & $\begin{array}{c}1 \\
(7.1 \%)\end{array}$ & $\begin{array}{c}11 \\
(78.6 \%)\end{array}$ & $\begin{array}{c}3 \\
(21.4 \%)\end{array}$ \\
\hline & $\begin{array}{l}4-6 \\
\text { hrs }\end{array}$ & $\begin{array}{c}40 \\
(50 \%)\end{array}$ & $\begin{array}{c}38 \\
(95 \%)\end{array}$ & $\begin{array}{c}2 \\
(5 \%)\end{array}$ & $\begin{array}{c}32 \\
(80 \%)\end{array}$ & $\begin{array}{c}6 \\
(15 \%)\end{array}$ & $\begin{array}{c}2 \\
(5 \%)\end{array}$ & $\begin{array}{c}17 \\
(42.5 \%)\end{array}$ & $\begin{array}{c}13 \\
(32.5 \%)\end{array}$ & $\begin{array}{c}10 \\
(25 \%)\end{array}$ & $\begin{array}{c}18 \\
(45 \%)\end{array}$ & $\begin{array}{c}14 \\
(35 \%)\end{array}$ & $\begin{array}{c}6 \\
(15 \%)\end{array}$ & $\begin{array}{c}2 \\
(5 \%)\end{array}$ & $\begin{array}{c}35 \\
(87.5 \%)\end{array}$ & $\begin{array}{c}5 \\
(12.5 \%)\end{array}$ \\
\hline & $>6 \mathrm{hrs}$ & $\begin{array}{c}24 \\
(30 \%)\end{array}$ & $\begin{array}{c}23 \\
(95.8 \%)\end{array}$ & $\begin{array}{c}1 \\
(4.2 \%)\end{array}$ & $\begin{array}{c}24 \\
(100 \%)\end{array}$ & $\begin{array}{c}0 \\
(0 \%)\end{array}$ & $\begin{array}{c}0 \\
(0 \%)\end{array}$ & $\begin{array}{c}17 \\
(70.8 \%)\end{array}$ & $\begin{array}{c}5 \\
(20.8 \%)\end{array}$ & $\begin{array}{c}2 \\
(8.3 \%)\end{array}$ & $\begin{array}{c}15 \\
(62.5 \%)\end{array}$ & $\begin{array}{c}8 \\
(33.3 \%)\end{array}$ & $\begin{array}{c}1 \\
(4.2 \%)\end{array}$ & $\begin{array}{c}0 \\
(0 \%)\end{array}$ & $\begin{array}{c}24 \\
(100 \%)\end{array}$ & $\begin{array}{c}0 \\
(0 \%)\end{array}$ \\
\hline \multicolumn{2}{|c|}{$\mathrm{P}$-value } & \multicolumn{3}{|c|}{0.965} & \multicolumn{3}{|c|}{0.043} & \multicolumn{3}{|c|}{0.143} & \multicolumn{4}{|c|}{0.442} & \multicolumn{2}{|c|}{0.158} \\
\hline \multirow{4}{*}{ 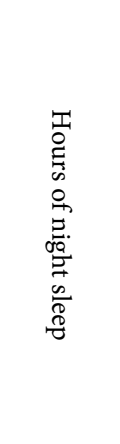 } & $<4 \mathrm{hrs}$ & $\begin{array}{c}10 \\
(12.5 \%)\end{array}$ & $\begin{array}{c}9 \\
(90 \%)\end{array}$ & $\begin{array}{c}1 \\
(10 \%)\end{array}$ & $\begin{array}{c}10 \\
(100 \%)\end{array}$ & $\begin{array}{c}0 \\
(0 \%)\end{array}$ & $\begin{array}{c}0 \\
(0 \%)\end{array}$ & $\begin{array}{c}7 \\
(70 \%)\end{array}$ & $\begin{array}{c}1 \\
(10 \%)\end{array}$ & $\begin{array}{c}2 \\
(20 \%)\end{array}$ & $\begin{array}{c}7 \\
(70 \%)\end{array}$ & $\begin{array}{c}2 \\
(20 \%)\end{array}$ & $\begin{array}{c}1 \\
(10 \%)\end{array}$ & $\begin{array}{c}0 \\
(0 \%)\end{array}$ & $\begin{array}{c}8 \\
(80 \%)\end{array}$ & $\begin{array}{c}2 \\
(20 \%)\end{array}$ \\
\hline & $\begin{array}{l}4 \text { - } 6 \\
\text { hrs }\end{array}$ & $\begin{array}{c}33 \\
(41.3 \%)\end{array}$ & $\begin{array}{c}33 \\
(100 \%\end{array}$ & $\begin{array}{c}0 \\
(0 \%)\end{array}$ & $\begin{array}{c}28 \\
(84.8 \%)\end{array}$ & $\begin{array}{c}5 \\
(15.2 \%)\end{array}$ & $\begin{array}{c}0 \\
(0 \%)\end{array}$ & $\begin{array}{c}19 \\
(57.6 \%)\end{array}$ & $\begin{array}{c}7 \\
(21.2 \%)\end{array}$ & $\begin{array}{c}7 \\
(21.2 \%)\end{array}$ & $\begin{array}{c}17 \\
(51.5 \%)\end{array}$ & $\begin{array}{c}13 \\
(39.4 \%)\end{array}$ & $\begin{array}{c}3 \\
(9.1 \%)\end{array}$ & $\begin{array}{c}0 \\
(0 \%)\end{array}$ & $\begin{array}{c}30 \\
(90.9 \%)\end{array}$ & $\begin{array}{c}3 \\
(9.1 \%)\end{array}$ \\
\hline & $\begin{array}{c}>6-8 \\
\text { hrs }\end{array}$ & $\begin{array}{c}22 \\
(27.5 \%)\end{array}$ & $\begin{array}{c}19 \\
(86.4 \% 0\end{array}$ & $\begin{array}{c}3 \\
(13.6 \%)\end{array}$ & $\begin{array}{c}16 \\
(72.7 \%)\end{array}$ & $\begin{array}{c}4 \\
(18.2 \%)\end{array}$ & $\begin{array}{c}2 \\
(9.1 \%)\end{array}$ & $\begin{array}{c}7 \\
(31.8 \%)\end{array}$ & $\begin{array}{c}10 \\
(45.5 \%)\end{array}$ & $\begin{array}{c}5 \\
(22.7 \%)\end{array}$ & $\begin{array}{c}9 \\
(40.9 \%)\end{array}$ & $\begin{array}{c}7 \\
(31.8 \%)\end{array}$ & $\begin{array}{c}3 \\
(13.6 \%)\end{array}$ & $\begin{array}{c}3 \\
(13.6 \%)\end{array}$ & $\begin{array}{c}20 \\
(90.9 \%)\end{array}$ & $\begin{array}{c}2 \\
(9.1 \%)\end{array}$ \\
\hline & $>8 \mathrm{hrs}$ & $\begin{array}{c}14 \\
(17.5 \%)\end{array}$ & $\begin{array}{c}14 \\
(100 \%)\end{array}$ & $\begin{array}{c}0 \\
(0 \%)\end{array}$ & $\begin{array}{c}11 \\
(78.6 \%)\end{array}$ & $\begin{array}{c}3 \\
(21.4 \%)\end{array}$ & $\begin{array}{c}0 \\
(0 \%)\end{array}$ & $\begin{array}{c}9 \\
(64.3 \%)\end{array}$ & $\begin{array}{c}2 \\
(14.3 \%)\end{array}$ & $\begin{array}{c}3 \\
(21.4 \%)\end{array}$ & $\begin{array}{c}4 \\
(28.6 \%)\end{array}$ & $\begin{array}{c}6 \\
(42.9 \%)\end{array}$ & $\begin{array}{c}4 \\
(28.6 \%)\end{array}$ & $\begin{array}{c}0 \\
(0 \%)\end{array}$ & $\begin{array}{c}13 \\
(92.9 \%)\end{array}$ & $\begin{array}{c}1 \\
(7.1 \%)\end{array}$ \\
\hline \multicolumn{2}{|c|}{ P-value } & \multicolumn{3}{|c|}{0.095} & \multicolumn{3}{|c|}{0.248} & \multicolumn{3}{|c|}{0.221} & \multicolumn{4}{|c|}{0.116} & \multicolumn{2}{|c|}{0.736} \\
\hline \multirow{5}{*}{ 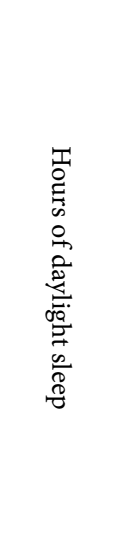 } & Never & $\begin{array}{c}3 \\
(3.8 \%)\end{array}$ & $\begin{array}{c}3 \\
(100 \%)\end{array}$ & $\begin{array}{c}0 \\
(0 \%)\end{array}$ & $\begin{array}{c}2 \\
(66.7 \%)\end{array}$ & $\begin{array}{c}1 \\
(33.3 \%)\end{array}$ & $\begin{array}{c}0 \\
(0 \%)\end{array}$ & $\begin{array}{c}1 \\
(33.3 \%)\end{array}$ & $\begin{array}{c}1 \\
(33.3 \%)\end{array}$ & $\begin{array}{c}1 \\
(33.3 \%)\end{array}$ & $\begin{array}{c}2 \\
(66.7 \%)\end{array}$ & $\begin{array}{c}0 \\
(0 \%)\end{array}$ & $\begin{array}{c}1 \\
(33.3 \%)\end{array}$ & $\begin{array}{c}0 \\
(0 \%)\end{array}$ & $\begin{array}{c}2 \\
(66.7 \%)\end{array}$ & $\begin{array}{c}1 \\
(33.3 \%)\end{array}$ \\
\hline & $<1 \mathrm{hrs}$ & $\begin{array}{c}6 \\
(7.5 \%)\end{array}$ & $\begin{array}{c}5 \\
(83.3 \%)\end{array}$ & $\begin{array}{c}1 \\
(16.7 \%)\end{array}$ & $\begin{array}{c}5 \\
(83.3 \%)\end{array}$ & $\begin{array}{c}1 \\
(16.7 \%)\end{array}$ & $\begin{array}{c}0 \\
(0 \%)\end{array}$ & $\begin{array}{c}4 \\
(66.7 \%)\end{array}$ & $\begin{array}{c}2 \\
(33.3 \%)\end{array}$ & $\begin{array}{c}0 \\
(0 \%)\end{array}$ & $\begin{array}{c}5 \\
(83.3 \%)\end{array}$ & $\begin{array}{c}0 \\
(0 \%)\end{array}$ & $\begin{array}{c}0 \\
(0 \%)\end{array}$ & $\begin{array}{c}1 \\
(16.7 \%)\end{array}$ & $\begin{array}{c}5 \\
(83.3 \%)\end{array}$ & $\begin{array}{c}1 \\
(16.7 \%)\end{array}$ \\
\hline & $\begin{array}{l}1 \text { - } 2 \\
\text { hrs }\end{array}$ & $\begin{array}{c}16 \\
(20 \%)\end{array}$ & $\begin{array}{c}16 \\
(100 \%)\end{array}$ & $\begin{array}{c}0 \\
(0 \%)\end{array}$ & $\begin{array}{c}11 \\
(68.8 \%)\end{array}$ & $\begin{array}{c}5 \\
(31.3 \%)\end{array}$ & $\begin{array}{c}0 \\
(0 \%)\end{array}$ & $\begin{array}{c}13 \\
(81.3 \%)\end{array}$ & $\begin{array}{c}1 \\
(6.3 \%)\end{array}$ & $\begin{array}{c}2 \\
(12.5 \%)\end{array}$ & $\begin{array}{c}5 \\
(31.3 \%)\end{array}$ & $\begin{array}{c}7 \\
(43.8 \%)\end{array}$ & $\begin{array}{c}4 \\
(25 \%)\end{array}$ & $\begin{array}{c}0 \\
(0 \%)\end{array}$ & $\begin{array}{c}15 \\
(93.8 \%)\end{array}$ & $\begin{array}{c}1 \\
(6.3 \%)\end{array}$ \\
\hline & $\begin{array}{c}>2-4 \\
\text { hrs }\end{array}$ & $\begin{array}{c}26 \\
(32.5 \%)\end{array}$ & $\begin{array}{c}26 \\
(100 \%)\end{array}$ & $\begin{array}{c}0 \\
(0 \%)\end{array}$ & $\begin{array}{c}23 \\
(88.5 \%)\end{array}$ & $\begin{array}{c}2 \\
(7.7 \%)\end{array}$ & $\begin{array}{c}1 \\
(3.8 \%)\end{array}$ & $\begin{array}{c}11 \\
(42.3 \%)\end{array}$ & $\begin{array}{c}7 \\
(26.9 \%)\end{array}$ & $\begin{array}{c}8 \\
(30.8 \%)\end{array}$ & $\begin{array}{c}16 \\
(61.5 \%)\end{array}$ & $\begin{array}{c}7 \\
(26.9 \%)\end{array}$ & $\begin{array}{c}2 \\
(7.7 \%)\end{array}$ & $\begin{array}{c}1 \\
(3.8 \%)\end{array}$ & $\begin{array}{c}24 \\
(92.3 \%)\end{array}$ & $\begin{array}{c}2 \\
(7.7 \%)\end{array}$ \\
\hline & $>4 \mathrm{hrs}$ & $\begin{array}{c}28 \\
(35 \%)\end{array}$ & $\begin{array}{c}25 \\
(89.3 \%)\end{array}$ & $\begin{array}{c}3 \\
(10.7 \%)\end{array}$ & $\begin{array}{c}24 \\
(85.7 \%)\end{array}$ & $\begin{array}{c}3 \\
(10.7 \%)\end{array}$ & $\begin{array}{c}1 \\
(3.6 \%)\end{array}$ & $\begin{array}{c}13 \\
(46.4 \%)\end{array}$ & $\begin{array}{c}9 \\
(32.1 \%)\end{array}$ & $\begin{array}{c}6 \\
(21.4 \%)\end{array}$ & $\begin{array}{c}9 \\
(32.1 \%)\end{array}$ & $\begin{array}{c}14 \\
(50 \%)\end{array}$ & $\begin{array}{c}4 \\
(14.3 \%)\end{array}$ & $\begin{array}{c}1 \\
(3.6 \%)\end{array}$ & $\begin{array}{c}25 \\
(89.3 \%)\end{array}$ & $\begin{array}{c}3 \\
(10.7 \%)\end{array}$ \\
\hline \multicolumn{2}{|c|}{$\mathrm{P}$-value } & & 0.2 & 204 & & 0.620 & & & 0.279 & & & & 112 & & 0.6 & \\
\hline
\end{tabular}


Table 5. Effects of selected eating habits on blood lipid profile among male Health Sciences students at Taif University, KSA.

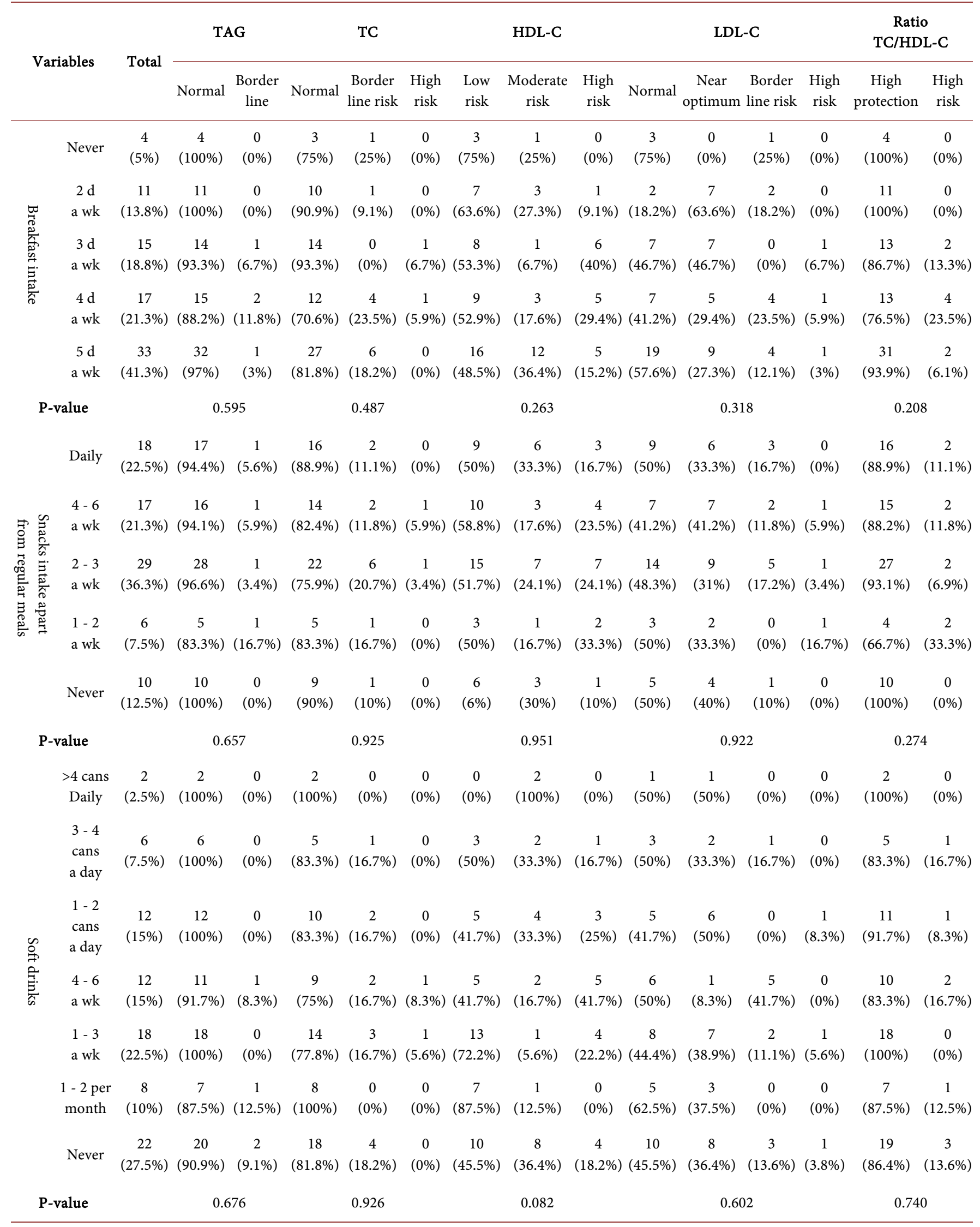


Table 6. Effects of selected food intake habits on blood lipid profile among male Health Sciences students at Taif University, KSA.

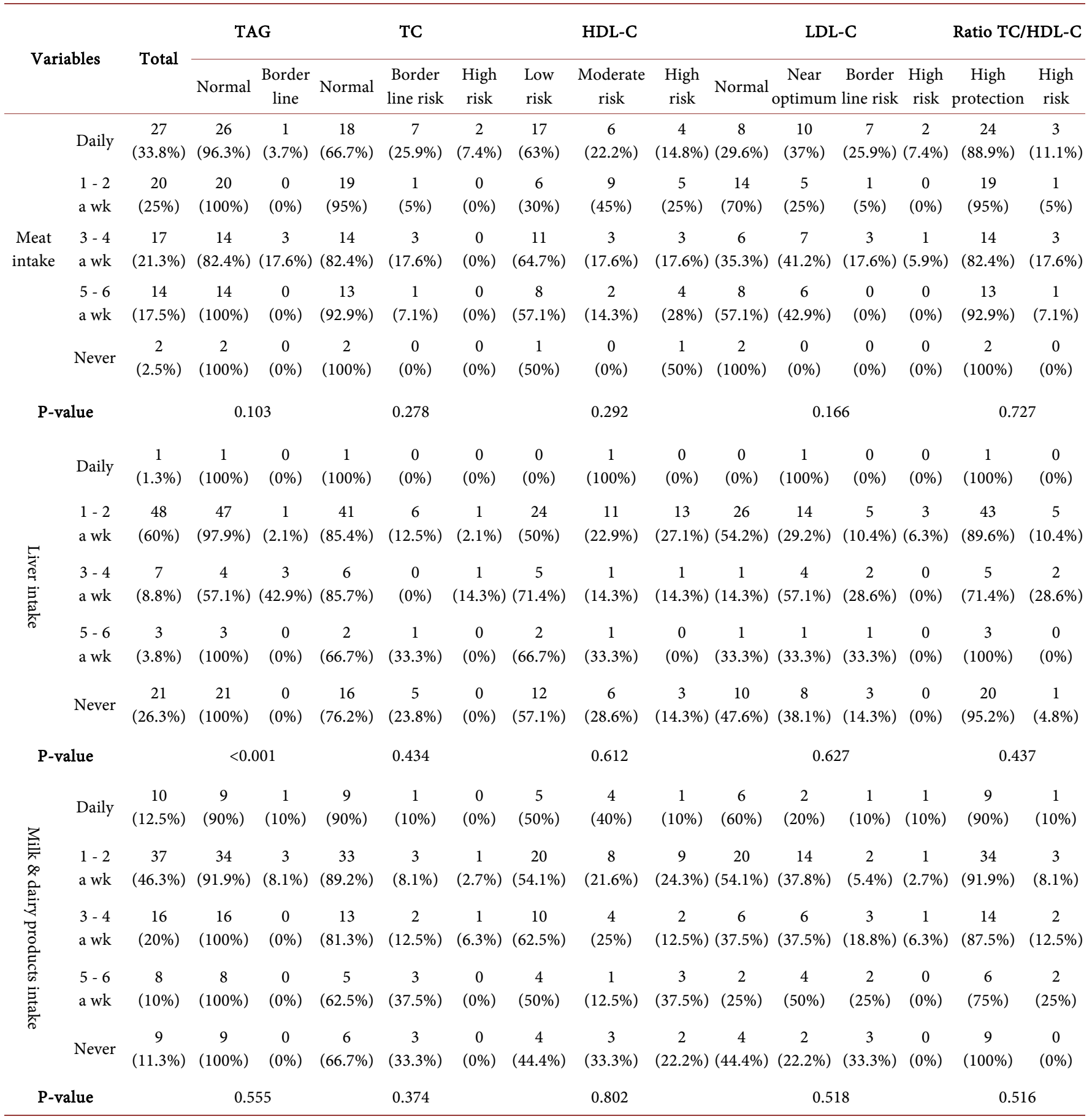

based studies, hypercholesterolemia rate (17.7\%) of the present study was much lower than in Turkish adults (37.5\%) [17], Spanish adults (24.3\%) [18], Saudi adults (43.3\%) [19], Jordanian adults (48.8\%) [20]. Furthermore, our figures are encouraging when compare them to the prevalence of hypercholesterolemia (36.7\%) among world's adults aged from 15 to 65 years old [12]. Hypercholesterolemia rate (17.7\%) among HSS reported in this study was very close to the prevalence of high blood cholesterol (17.0\%) in American adults [21]. 
An interesting finding of the present study was related to the high prevalence of low HDL-c. Results showed that $46.3 \%$ of male university students had HDL-c levels in the moderate to high risk values. This is much higher than figures reported in other studies on this age group [10] [14] [16]. The prevalence of elevated LDL-c (130 mg/dl and above) (16.8\%) among Taif University students was much lower than their counterparts in Egypt (33.1\%) [10] and in Iraq (30\%) [14].

The overall prevalence of dyslipidemia was $60.0 \%$, which agreed with the findings of Abdel Wahed et al. (63.8\%) [10], and Al Sabah et al. (75\%) [14]. On the other hand, dyslipidemia rate reported in this study was much higher than its rate in a Kuwaiti university (10.5\%) [16]. Dyslipidemia (especially low HDL-c) reported in the present study was considerably linked with overweight/obesity. This is consistent with other studies [10] [11] [14] [22]. Overweight/obesity was associated with other blood lipid parameters but this link was statistically insignificant. Considerable relationship between abdominal adiposity and low HDL-c level was noted, which agreed with findings of other studies [10] [17]. Bibiloni et al. [18] found that adolescents with abdominal obesity had the highest rate of dyslipidemia, mostly high TAG.

The relationship between socio-demographic variables and dyslipidemia among HSS indicated that just academic level was associated with low HDL-c levels, but the remaining socio-demographic factors (living with family, family income, and location of residency) were associated with abnormal lipid profile but this link was statistically insignificant. Abdel Wahed et al. [10] found increased dyslipidemia rate among university students of medicine, computer sciences and information. The highest low HDL-c levels (69.3\%) were noted for the refreshment students, which could be related to a significant weight gain during the first year. Many investigations showed that certain periods of our lives that are more likely to influence lifestyle and thus body mass [23]. For example, the transition from secondary school to university appears to be associated with a reduction in physical activities, an increase in deskbound activates, therefore, students gain weight [24]. The term "freshman 15" means that students in their 1st year gain 15 pounds. It is well known that obesity is associated with dyslipidemia. The current study has reported that one in every two students is either overweight and/or obese. This high rate of obesity and overweight could be the main cause of high dyslipidemia of the present study.

Significant association was noted between hours of TV viewing/use of computers/ playing video games and high TC. There is a negative association between hours of TV watching and physical activities. This means a reduction in physical activities and an increase in sedentary activities. Several intervention studies have shown enhancements in blood lipid chemistry with increase in physical activities [17] [25]. Results of this study are consistent with similar studies [10] [17] [26].

\section{Conclusion}

This is the first human study conducted at Taif University. This study combined different tools for data collection: laboratory, anthropometry and questionnaire. The overall prevalence of dyslipidemia was $60.0 \%$.The prevalence of hypercholesterolemia, hypertriglyceridemia, high LDL-c, and low HDL-c was $17.7 \%, 5.0 \%, 16.8 \%$, and $46.3 \%$, 
respectively. Low HDL-c levels were the main prevalent dyslipidemia among participants. Overweight/obese students were more likely to show at least one undesirable lipid concentration. BMI, W_C, and academic level were significantly associated with unacceptable levels of HDL-c. TV viewing had significant effects on hypercholesterolemia, while only liver intake had significant impact on high TAG. This study recommends creation of health awareness plan to educate university students concerning healthy lifestyle and healthy food options. Special attention should be directed toward increase physical activities, decrease times spent on TV viewing, playing video games, using computers. It also recommends a decrease in liver intake and to increase foods that improve good cholesterol (HDL-c). Since our study population is relatively small and limited to the Health Sciences male university students, conclusions from the present study could not be generalized further. Further investigations using modern technologies for assessment of cardio-metabolic risk factors are needed. Additionally, further in-depth community based studies are recommended in order to plan an effective control program.

\section{Acknowledgements}

Author of this study conveys sincere thanks to students who kindly agreed to participate in this study. I thank Dr. Ahmed S. Eldalo, and students: Adnan A. Alnofeie, Waleed Y. Alghamdi, Salman S. Almutairi, Fahd S. Badyan for their valuable contribution in data collection. Special thanks are conveyed to Elaj Laboratories, Jeddah, KSA for their help in conducting lipid profile analysis.

\section{Conflict of Interest}

The author of this study declares no conflict of interest

\section{Ethical Consideration}

This study was ethically approved by the Research Ethical Committee at Taif University, KSA (No. 85310). Each participant carefully read and signed a written consent form before filling the questionnaire or withdrawing blood sample.

\section{References}

[1] Al Balla, S.R., Bamgboye, E.A., Sekait, M. and Balla, M. (1993) Causes of-Morbidity in the Elderly Population of Saudi Arabia. Journal of Tropical Medicine and Hygiene, 96, 157-162.

[2] Al Balla, S.R., Bamgboye, E.A., Al Balla, S.R., Al Sekait, M. and Al Rasheed, R. (1993) Pattern of Adult Admission into Medical Wardsof King Khalid University Hospital, Riyadh (1985-1990). Saudi Medical Journal, 13, 8-13.

[3] Smith, G.D. and Ebrahim, S. (2001) Is It Time to Call it a Day? International Journal of Epidemiology, 30, 1-11. https://doi.org/10.1093/ije/30.1.1

[4] Al-Nuaim, A.R., Al-Rubeaan, K., Al-Mazroub, Y., Al-Attas, O. and Al-Daghari, N. (1996) Prevalence of Hypercholesterolemia in Saudi Arabia, Epidemiological Study. International Journal of Cardiology, 54, 41-49. https://doi.org/10.1016/0167-5273(96)02555-7

[5] Manios, Y., Moschandreas, J., Hatzis, C. and Kafatos, A. (2002) Health and Nutrition Education in Primary Schools of Crete: Changes in Chronic Disease Risk Factors Following a 6Year Intervention Programme. British Journal of Nutrition, 88, 315-324. 
https://doi.org/10.1079/BJN2002672

[6] Al-Shehri, S.N., Saleh, Z.A., Salama, M.M. and Hassan, Y.M. (2004) Prevalence of Hyperlipidemia among Saudi School Children in Riyadh. Annals of Saudi Medical Journal, 24, 6-8.

[7] Al-Nuaim, A.R., Al-Rubeaan, K., Al-Mazroub, Y., Al-Attas, O. and Al-Daghari, N.(1997) Serum Total and Fractionated Cholesterol Distribution and Prevalenceof Hypercholesterolemia in Urban and Rural Communities in Saudi Arabia. International Journal of Cardiology, 58, 141-149. https://doi.org/10.1016/S0167-5273(96)02850-1

[8] American Heart Association (2016) Understand Your Risk for High Cholesterol. Dallas. http://www.heart.org/HEARTORG/Conditions/Cholesterol/Cholesterol UCM $001089 \mathrm{Su}$ bHomePage.jsp

[9] Kuczmarski, R.J., Ogden, C.L., Guo, S.S., et al. (2002) CDC Growth Charts for the United States: Methods and Development. National Center for Health Statistics. Vital Health Statistics, 11, 1-147.

[10] Abdel Wahed, W.Y., El-Khashab, K. and Hassan, S.K. (2016) Prevalence of Dyslipidaemia among Healthy University Students: Fayoum Governorate, Egypt. Epidemiology Biostatistics Public Health Journal, 13, E11769-1-E11769-9.

[11] Desouky, D.S., Omar, M.S., Nemenqani, D.M., Jabbar, J. and Tarak-Khan, N.M. (2004) Risk Factors of Non-Communicable Diseases among Female University Students of the Health Colleges of Taif University. International Journal of Medical Sciences, 6, 97-107.

[12] WHO and ARE-Ministry of Health Population (2011-2012) Egypt National STEPwise Survey of Non Communicable Diseases Risk Factors.

[13] Sabra, A.A., Attia, Z., Al-Sebiany, A.M., Al-Kurashi, N.Y. and Al-Zubier, A.G. (2009) Coronary Heart Disease Risk Factors: Prevalence and Behavior among Male University Students in Dammam City, Saudi Arabia. The Journal of the Egyptian Public Health Association, 82, 21-42.

[14] Al-Sabah, H.A., Hussain, N.H. and Ali, D.T. (2014) Dyslipidemia in Young Adults Aged (20-40) Years Attending Baghdad Teaching Hospital and Al-Mansour Primary Health Care Center in Baghdad City. The Iraqi Postgraduate Medical Journal, 13, 320-327.

[15] Shawar, S.M., Al-Bati, N.A., Al-Mahameed, A., Nagalla, D.S. and Obeidat, M. (2012) Hypercholesterolemia among Apparently Healthy University Students. Oman Medical Journal, 27, 274-280. https://doi.org/10.5001/omj.2012.69

[16] AlMajed, H.T., Al Attar, A.T., Sadek, A.A., AlMuaili, T.A., AlMutairi, O.A. and Al Torah, W.A. (2011) Prevalence of Dyslipidemia and Obesity among College Students in Kuwait. Alexandria Journal of Medicine, 47, 67-71. https://doi.org/10.1016/j.ajme.2010.12.003

[17] Erem, C., Hacihasanoglu, A., Deger, O., Kocak, M. and Topbas, M. (2008) Prevalence of Dyslipidemia and Associated Risk Factors among Turkish Adults: Trabzon Lipid Study. Endocrine, 34, 36-51. https://doi.org/10.1007/s12020-008-9100-Z

[18] Bibiloni, M.M., Salas, R., Pons, A. and Tur, J.A. (2015) Prevalence of Dyslipidaemia and Associated Risk Factors among Balearic Islands Adolescents, a Mediterranean Region. European Journal of Clinical Nutrition, 69, 722-728. https://doi.org/10.1038/ejen.2014.236

[19] Ogbeide, D.O., Karim, A., Al-Khalifa, I.M. and Siddique, S. (2004) Population Based Study of Serum Lipid Levels in Al-Kharj Health Center, Saudi Arabia. Saudi Medical Journal, 25, 1855-1857.

[20] Khader, Y.S., Batieha, A., El-Khateeb, M. and Al Omari M. (2010) Prevalence of Dyslipidemia and Its Associated Factors among Jordanian Adults. Journal of Clinical Lipidology, 4, 53-58. https://doi.org/10.1016/j.jacl.2009.12.004

[21] American Heart Association (AHA) https://www.cdc.gov/nchs/data/series/sr 11/sr11 156.pdf

[22] Al-Kaabba, A.F., Al-Hamdan, N.A., El Tahir, A., Abdalla, A.M., Saeed, A.A. and Hamza, 
M.A. (2012) Prevalence and Correlates of Dyslipidemia among Adults in Saudi Arabia: Results from a National Survey. Journal of Endocrine and Metabolic Diseases, 2, 89-97. https://doi.org/10.4236/ojemd.2012.24014

[23] Pe'russe-Lachance, E., Tremblay, A. and Drapeau, V. (2010) Lifestyle Factors and Other Health Measures in a Canadian University Community. Applied Physiology, Nutrition, and Metabolism, 35, 498-506. https://doi.org/10.1139/H10-035

[24] Crombie, A.P., Ilich, J.Z., Dutton, G.R., Panton, L.B. and Abood, D.A. (2009) The Freshman Weight Gain Phenomenon Revisited. Nutrition Review, 67, 83-94. https://doi.org/10.1111/j.1753-4887.2008.00143.x

[25] Kang, W.M., Zhang, J.S., Liu, X.X., Wang, M.S., Zhao, M.L. and Yu, J.C. (2009) Prevalence of Abnormity of Blood Lipid and Associated Factors in Health Examination Population in Beijing. Chinese Medical Sciences Journal, 24, 142-146. https://doi.org/10.1016/S1001-9294(09)60078-X

[26] Pagels, P., Raustorp, A., Archer, T., Lidman, U. and Alricsson, M. (2012) Influence of Moderate, Daily Physical Activity on Body Composition and Blood Lipid Profile in Swedish Adults. Journal of Physical Activity and Health, 9, 867-874.

https://doi.org/10.1123/jpah.9.6.867

\section{Submit or recommend next manuscript to SCIRP and we will provide best service for you:}

Accepting pre-submission inquiries through Email, Facebook, LinkedIn, Twitter, etc. A wide selection of journals (inclusive of 9 subjects, more than 200 journals)

Providing 24-hour high-quality service

User-friendly online submission system

Fair and swift peer-review system

Efficient typesetting and proofreading procedure

Display of the result of downloads and visits, as well as the number of cited articles

Maximum dissemination of your research work

Submit your manuscript at: http://papersubmission.scirp.org/

Or contact fns@scirp.org 